\title{
Improved Kidney Function Following Physiologic Insulin Resensitization Treatment Modality
}

\author{
${ }^{1}$ Island Doctors, St. Augustine, FL with Well Cell Global, Houston, TX. \\ ${ }^{2}$ Department of Developmental and Cell Biology, University of California Irvine, Irvine, CA. \\ ${ }^{3}$ Department of Surgery, University of California Irvine, Orange, CA. \\ ${ }^{4}$ Department of Biomedical Engineering, University of California Irvine, Irvine, CA.
}

Zach Villaverde' ${ }^{\text {, Tori Tucker }}{ }^{2}$, Michael Alexander ${ }^{3}$, Scott A. Hepford ${ }^{1}$, Jonathan RT Lakey ${ }^{3 *}$, Roy H. Hinman II1 ${ }^{1}$

*Corresponding Author: Jonathan Lakey, Professor, Departments of Surgery and Biomedical Engineering University of California Irvine.

Received Date: 07 July 2021 | Accepted Date: 28 July 2021 | Published Date: 30 July 2021

Citation: Z Villaverde, T Tucker, M Alexander, Scott A. Hepford, JRT Lakey, et al. (2021) Improved Kidney Function Following Physiologic Insulin Resensitization Treatment Modality. J. Endocrinology and Disorders. 5(4): DOI:10.31579/2640-1045/080

Copyright: @ 2021 Jonathan Lakey. This is an open-access article distributed under the terms of the Creative Commons Attribution License, which permits unrestricted use, distribution, and reproduction in any medium, provided the original author and source are credited.

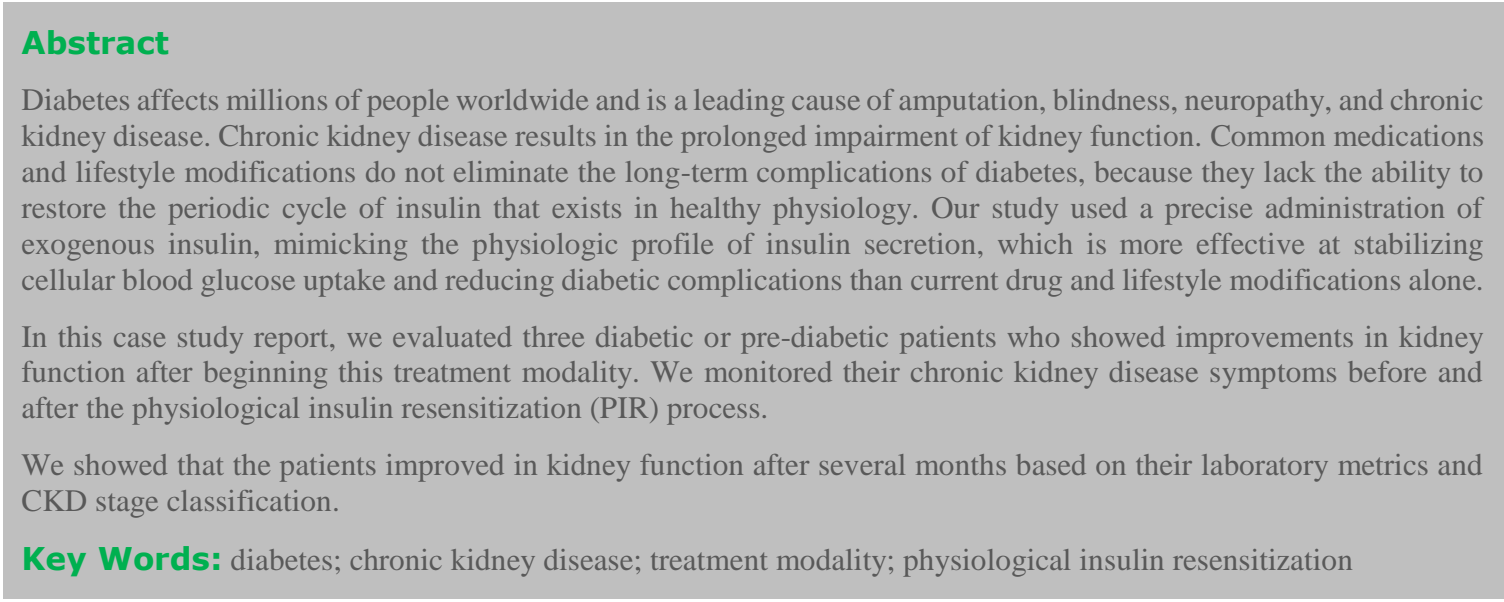

\section{Introduction}

Diabetes mellitus affects millions of people worldwide, and its complications cost hundreds of billions of dollars to treat. Diabetes is a metabolic disorder that affects the way humans metabolize glucose [1]. Glucose is of vital importance to human health, as it is the main energy molecule for muscles and organs such as the brain. Diabetes is typically identified via sustained hyperglycemia [1]. This hyperglycemia is caused by at least one defect along the insulin secretion and reception pathway in the body [1].

The two most common types of diabetes are Type 1 and Type 2 diabetes [1]. Type 1 diabetes (T1D) is an autoimmune disease that results in the immune system destroying insulin-producing beta cells in the pancreas. T1D typically manifests itself in early adolescence, and T1D patients are dependent on exogenous insulin for survival [1]. Type 2 diabetes (T2D) is the more common of the two and is typically believed to be caused by inadequate insulin secretion from the pancreas or decreased cellular response to insulin [1]. Onset of T2D is typically believed to be associated with obesity or poor diet [1]. In people developing T2D, hyperglycemia is caused by either a decrease in insulin sensitivity, a failure to produce enough insulin or both. In the case of decreased insulin sensitivity, abnormal insulin secretion from the pancreas results in consistent exposure of cells to insulin. Cells consequently downregulate the associated insulin receptors, and the patient takes up less glucose into his or her cells, prompting the pancreas to produce more insulin in a positive feedback loop [1]. Eventually, the patient has so few responsive receptors that glucose cannot be taken up into cells, and the patient suffers from chronic hyperglycemia [1]. In the case of insulin deficiency, genetic defects or chronic overproduction of insulin can result in not enough insulin being secreted from the pancreas. In this case, hyperglycemia is sustained because a patient does not produce enough insulin for the cells to uptake the glucose in the bloodstream [1]. Both of the above scenarios result in T2D characterized by hyperglycemia, which can lead to other detrimental effects on the body.

One potential effect of diabetes is increased inflammatory response. Inflammation has been hypothesized to originate from decreased insulin 
sensitivity, as well as obesity and increased lipolysis [2]. Insulin has been shown to suppress certain inflammation-promoting transcription factors such as IL-6, CRP, and TNF- $\alpha$. Diabetes can decrease production or uptake of insulin, which removes the suppression of these transcription factors and results in inflammation [2]. Type 2 diabetics can develop obesity because their blood sugar is not being effectively metabolized; thus, the body stores the blood sugar as fat before it can be utilized through the fat metabolism pathway [2]. Obesity results in increased numbers of adipocytes, which constitutively express inflammatory markers, like IL-6 and CRP as mentioned above [2]. Finally, increased lipolysis because of T2D results in higher free fatty acid concentrations in the blood, which contributes to increased cellular oxidative stress through the buildup of oxygen radicals, and tissue inflammation [2]. The combination of sustained hyperglycemia and inflammation in the body as a result of diabetes contributes to many of the complications that arise in diabetic patients [2].

Many complications can arise from chronic hyperglycemia and inflammation associated with diabetes and other metabolic disorders. Some of the more common complications include cardiovascular disease, wound ulceration, peripheral neuropathy in limbs, and nephropathy leading to chronic kidney disease (CKD) and kidney failure [3]. Chronic kidney disease is of growing concern, as the number of people per year initiating treatment for diabetic CKD leading to end-stage kidney disease (ESKD) and dialysis increased more than 18-fold between 1980 and 2011, with numbers continuing to climb [3]. Diabetic CKD, also known as diabetic kidney disease (DKD), is also the single strongest predictor of mortality in diabetic patients, warranting it special attention in medicine [3].

Chronic kidney disease is the result of prolonged impairment of kidney function. Kidney function is best measured through a patient's estimated Glomerular Filtration Rate, or (e) GFR [4]. Clinically, a patient is diagnosed with CKD if he or she exhibits one or both of the following for at least three months: an eGFR of below $60 \mathrm{~mL} / \mathrm{min}$ or at least one other marker of kidney damage [4]. These markers include albuminuria or proteinuria, abnormal urine electrolyte levels, structural abnormalities in the kidney, and others [4]. Diabetic patients are at unique risk for developing CKD because two of the side effects of diabetes, hyperglycemia and inflammation, affect pathways that lead to impaired kidney function [3]. Hyperglycemia changes the typical progression of glycolysis in cells, resulting in upregulation of transcription factors and substrates that lead to glomerular hyperfiltration or capillary obstruction. Over time, hyperfiltration and blood vessel obstruction damage the kidneys and can result in DKD [3]. Inflammation results in increased levels of inflammatory cytokines in the blood, which are hypothesized to increase endothelial cell permeability as well as induce apoptosis in endothelial cells. Degradation of endothelial linings can contribute to abnormal amounts of protein or electrolytes in urine, as well as decreased eGFR in damaged nephrons [3].

Chronic kidney disease is generally separated into six stages based on a patient's eGFR. Stage 1 CKD is "normal" and is characterized as an eGFR of $90 \mathrm{~mL} / \mathrm{min}$ or higher. Stage $2 \mathrm{CKD}$ is within the range of $60 \mathrm{~mL} / \mathrm{min}$ to $89 \mathrm{~mL} / \mathrm{min}$, and kidneys are considered mildly impaired. Patients with an eGFR below $60 \mathrm{~mL} / \mathrm{min}$ are then diagnosed with CKD and placed into CKD stage 3 . Stage 3 is further divided into $3 a$ and $3 b$ and signals moderate impairment in kidney function. Stage 3 a falls within eGFR ranges of $45 \mathrm{~mL} / \mathrm{min}$ to $59 \mathrm{~mL} / \mathrm{min}$ and stage $3 \mathrm{~b}$ within $30 \mathrm{~mL} / \mathrm{min}$ and $44 \mathrm{~mL} / \mathrm{min}$. CKD Stage 4 denotes significant impairment and risk for renal failure. Stage 4 eGFRs range from $15 \mathrm{~mL} / \mathrm{min}$ to $29 \mathrm{~mL} / \mathrm{min}$. Below $15 \mathrm{~mL} / \mathrm{min}$ is CKD Stage 5, which is essentially renal failure [4]. Patients at CKD Stage 5 are highly likely to require dialysis or a kidney transplant [4]. Dialysis and surgery can be costly and significantly impact a patient's quality of life. Current widely accepted treatments focus on slowing the progression of CKD and attempt to restore "reversible" factors of CKD to normal to reduce its severity [5]. Once a diabetic patient begins to lose kidney function, however, it is extremely difficult to arrest this progression, because the underlying contributors, high blood sugar and inflammatory tissue damage, are still present in the patient. This study seeks to explore the effect of Physiologic Insulin Resensitization on diabetic and pre-diabetic patients in varying stages of CKD. More specifically, we want to determine whether physiologic insulin resensitization displayed any noticeable effects on the kidney function of patients currently receiving the treatment modality in a real-world clinical setting.

\section{Description of Physiologic Insulin Resensitization (PIR)}

In our study, we utilize a novel therapeutic protocol that uses insulin as a biologic adjunct in treating the systemic complications of diabetes regarding kidney function. The advantage of this protocol is mimicking the body's own method of insulin regulation, especially mimicking the normal physiologic and periodic insulin signaling. This protocol was designed to counter the negative effect of insulin messaging, as well as unopposed glucagon effect on reducing insulin receptor expression [2]. Effectively, this protocol serves as a potent adjunctive companion method to overcome insulin resistance, or insulin toxicity and improve carbohydrate metabolism.

This bio-mimicry precision dosing ranges dynamically between 4- to 8minutes intervals where adjustments to concentrations, volumes, pressures, and oscillations of insulin are varied on an individualized basis. Oral glucose is used at individualized intervals to trigger carbohydrate metabolism, which as carbohydrate metabolism improves, ATP production is increased, and inflammatory markers are reduced, leading to higher available cellular energy [7].

The PIR precision dosing pattern of insulin is consistent with normal hormone secretion and more closely approximates the body's natural signaling pathways. As the patient's insulin resistance improves, healthcare providers can titrate other medications to optimize treatment regimens. In general, PIR permits lowering dosing of subcutaneous insulin and other diabetes medications, which often promote the secretion of insulin or inhibit the production of glucose and are costly [7]. This study was performed as an observational evaluation of patients who are already undergoing PIR treatment at Island Doctors and have their kidney function monitored as a part of the treatment.

\section{Consent for study}

Before any patient begins the PIR treatment modality at the Island Doctors clinic in Palm Coast, Florida, the patient signs a consent document to their information being used anonymously for the purpose of scientific studies. Patient data shown in this manuscript complies with the terms of this agreement.

\section{Clinical Presentation}

Tables 1-3 are results from three male patients who have been on PIR infusion treatment for at least five weeks. These three patients were selected from more than 80 current patients for exceptional changes in metrics related to kidney function. Table 1 identifies patients based on their ID number at the clinic where they receive their PIR infusion treatments. Their ages are also noted. These patients range from 75 at the youngest to 85 at the oldest. It is worth noting that only one of the patients, patient 8 , was referred to the PIR clinic for CKD stage 3 . The other two patients were referred for other reasons relating to PIR. Only patient 32 is clinically diabetic. The other two patients are pre-diabetic, but all three of them have low eGFR scores that would classify them as CKD stage 3 or higher. 


\begin{tabular}{|c|c|c|}
\hline Patient ID & Age & Reason for Starting the PIR Treatment Modality \\
\hline 8 & 77 & CKD Stage 3/Peripheral Neuropathy \\
\hline 30 & 75 & Ulcerated Wound \\
\hline 32 & 85 & Diabetic Neuropathy \\
\hline
\end{tabular}

Table 1: Patient characteristics enrolled in this study. Basic demographic information as well as the reason for referral to the PIR treatment modality is also shown.

Table 2 outlines the relevant metrics for assessment of patient kidney function. Each of the three patients observed in this case study was on the treatment modality for at least 5 weeks prior to re-evaluation of these metrics. This translates to a minimum of 8-10 infusions for patient 32 , and approximately 20 for patients 8 and 30 after 15 weeks of the treatment modality. The primary indicator for CKD, eGFR, showed a positive percent change between the base and the first test for each of the three patients. Patient 8 showed the highest percentage increase of $67 \%$, but all three of these patients increased at least $25 \%$ in this short window of treatments. Furthermore, patient 8 regressed classification from CKD stage $3 \mathrm{~b}$ to $3 \mathrm{a}$, and patient 30 regressed from $3 \mathrm{a}$ to 2 . These patients additionally showed decreases in both BUN and Creatinine in their urinalysis tests. The patients' BUN scores decreased anywhere from $21 \%$ to $46 \%$ over this timeframe, and Creatinine decreased between $18 \%$ and $34 \%$. These changes are all positive developments in kidney health of these patients.

\begin{tabular}{|c|c|c|c|c|c|c|c|c|c|c|}
\hline $\begin{array}{l}\text { Patient } \\
\text { ID } \\
\end{array}$ & Lab Date & $\begin{array}{l}\text { Weeks on } \\
\text { Infusion } \\
\text { Treatment }\end{array}$ & $\begin{array}{l}\text { BUN } \\
(\mathrm{mg} / \mathrm{dL})\end{array}$ & $\%$ Chg & $\begin{array}{l}\text { Creatinine } \\
(\mathrm{mg} / \mathrm{dL})\end{array}$ & $\%$ Chg & $\begin{array}{l}\text { eGFR } \\
(\mathrm{mL} / \mathrm{min})\end{array}$ & $\%$ Chg & $\begin{array}{l}\text { Potassium } \\
(\mathrm{mmol} / \mathrm{L})\end{array}$ & $\%$ Chg \\
\hline 8 & $9 / 19 / 2020$ & 0 & 28 & & 1.9 & & 33 & & 5.1 & \\
\hline 8 & $1 / 12 / 2021$ & 15 & 15 & $-46 \%$ & 1.25 & $-34 \%$ & 55 & $67 \%$ & 4.7 & $-8 \%$ \\
\hline 30 & $8 / 27 / 2020$ & 0 & 19 & & 1.3 & & 53.8 & & 3.8 & \\
\hline 30 & $1 / 13 / 2021$ & 15 & 15 & $-21 \%$ & 0.99 & $-24 \%$ & 74 & $38 \%$ & 4 & $5 \%$ \\
\hline 32 & $9 / 17 / 2020$ & 0 & 35 & & 1.81 & & 33 & & 5.9 & \\
\hline 32 & $1 / 18 / 2021$ & 5 & 19 & $-46 \%$ & 1.49 & $-18 \%$ & 42 & $27 \%$ & 4.3 & $-27 \%$ \\
\hline
\end{tabular}

Table 2: Kidney lab markers before and after beginning physiologic insulin resensitization for kidney function. Relevant metrics for kidney function as well as percent changes from baseline are outlined for each patient. Green labels indicate strictly positive developments in percent change. The date of the baseline labs - 0 weeks on the treatment modality - does not correspond with the first day of patient treatment. These dates correspond to the patients' most recent labs prior to beginning the treatment modality. The number of weeks on infusion treatments is tabulated separately.

Another development to note is the potassium level in patient 32. At the beginning of this treatment modality, this patient showed a potassium level of $5.9 \mathrm{mmol} / \mathrm{L}$, which is higher than the normal upper boundary of 5.2. After 5 weeks of treatments, this patient showed a potassium of 4.3 $\mathrm{mmol} / \mathrm{L}$, decreasing $27 \%$, and falling back within normal levels. Likewise, patient 8 showed potassium of $5.1 \mathrm{mmol} / \mathrm{L}$, which is almost at the upper limit of normal levels. After 15 weeks of treatments, that number decreased $8 \%$ to $4.7 \mathrm{mmol} / \mathrm{L}$.
In terms of diabetes-specific results, Table 3 shows the change in each patient from their baseline labs. For the three patients, fasting blood glucose gave ambiguous results, with one patient decreasing, one patient increasing, and one staying roughly the same. However, Hemoglobin A1c did decrease in all three patients, even those who are not clinically diabetic. Hemoglobin A1C decreased most drastically in patient 32, the one patient of these three who is clinically diabetic, decreasing a full percentage point from $7.3 \%$ to $6.3 \%$.

\begin{tabular}{|l|l|l|l|l|l|l|}
\hline \multicolumn{6}{|c|}{ Table 3: Diabetes Specific Metrics by Patient - Before and During the PIR Treatment Modality } \\
\hline Patient ID & Lab Date & $\begin{array}{l}\text { Weeks on Infusion } \\
\text { Treatment }\end{array}$ & $\begin{array}{l}\text { Fasting BG } \\
(\mathrm{mg} / \mathrm{dL})\end{array}$ & $\%$ Chg & $\begin{array}{l}\text { A1C } \\
(\%)\end{array}$ & $\%$ Chg \\
\hline 8 & $9 / 19 / 2020$ & 0 & 116 & & 6.3 & \\
\hline 8 & $1 / 12 / 2021$ & 15 & 100 & $-14 \%$ & 6 & $-5 \%$ \\
\hline 30 & $8 / 27 / 2020$ & 0 & 94 & & 5.7 & \\
\hline 30 & $1 / 13 / 2021$ & 15 & 96 & $2 \%$ & 5.6 & $-2 \%$ \\
\hline 32 & $9 / 17 / 2020$ & 0 & 100 & & 7.3 & \\
\hline 32 & $1 / 18 / 2021$ & 5 & 142 & $42 \%$ & 6.3 & $-14 \%$ \\
\hline
\end{tabular}

Table 3: Diabetic lab markers before and after beginning physiologic insulin resensitization. Relevant metrics for diabetes as well as percent changes from baseline are outlined for each patient. Green labels indicate strictly positive developments in percent change. This information corresponds to the same rows/columns as Table 2 


\section{Discussion}

The link between insulin resistance and progressive CKD is becoming apparent as more research shows physiologic effects on the kidneys of insulin resistance. A 2012 study by the American Society of Nephrology noted insulin resistance as a known complication of ESRD. [8] The study went on to show an inverse relation between insulin resistance and measured eGFR, which is one of the key metrics in determining severity of CKD. [8,9]. Diabetic patients suffer from insulin resistance with or without CKD and ESRD, but this study also notes that insulin resistance is prevalent in non-diabetic ESRD patients as well. [8]

The goal of the PIR treatment is to return insulin secretion to normal physiological conditions, thereby reducing the level of insulin resistance in affected patients. The patients sampled in the results above display increased eGFR, which is correlated with reduced insulin resistance in both the diabetic and nondiabetic patients. This is potentially attributable to the PIR treatment.

Insulin resistance has also been shown to create structural changes in the kidney, including excess proliferation of cells, apoptosis inhibition, and reduction in endothelial functionality [6]. All these effects carry a risk of kidney injury and progressive CKD [6]. The PIR modality recalibrates the endogenous production of insulin to its physiologic "pulse-rest" intervals, which may allow these alternative insulin-signaling pathways to regain ordinary functionality. This may result in a restructuring of kidney tissues back to normal, and furthermore an improvement in kidney function after beginning the PIR treatment modality.

\section{Conclusion}

The principal objective of this study was to determine if the PIR treatment modality has any noticeable effect on kidney function in patients with diabetic CKD. Given the above results, it is possible that this modality has positive effects on kidney function in diabetic patients. Because the study was a real-world analysis and not a controlled study with explicit variables, further data and studies are required to support this notion. The next steps after this preliminary case study would be design and execution of a full controlled study among diabetic patients with CKD, in which the independent variable is the PIR treatment modality itself.

\section{Funding}

This work was supported by internal funding by Well Cell Global LLC and volunteered time by providers and staff members at Island Doctors.

\section{Declaration of Interests}

Dr. Roy Hinman, and Zach Villaverde are employees of Island Doctors, which administers the PIR treatment modality. Scott Hepford is employed by Well Cell Global LLC, which researches, advances and licenses the PIR treatment modality. Tori Tucker, Michael Alexander, and Dr. Jonathan Lakey are employees of the University of California Irvine and have no conflicts of interest in this study.

\section{Consent for Publication}

Patients provided their consent for anonymous inclusion of their data in publications.

\section{References}

1. American Diabetes Association. (2005) Diagnosis and Classification of Diabetes Mellitus. Diabetes Care. 28(supply 1):s37-s42.

2. Paresh D, Ahmad A, Ajay C, Priya M, Rajesh G. (2005) Metabolic Syndrome. Circulation.111 (11):1448-1454.

3. Toth-Manikowski S, Atta MG. (2015) Diabetic Kidney Disease: Pathophysiology and Therapeutic Targets. J Diabetes Res. e697010.

4. Webster AC, Nagler EV, Morton RL, Masson P. (2017) Chronic Kidney Disease. The Lancet. 389(10075):1238-1252.

5. Johnson CA, Levey AS, Coresh J, Levin A, Lau J, Eknoyan G. (2004) Clinical Practice Guidelines for Chronic Kidney Disease in Adults: Part 1. Definition, Disease Stages, Evaluation, Treatment, and Risk Factors. Am Fam Physician. 70(5):869876.

6. Abernethy A. (2020) Insulin Gains New Pathway to Increased Competition.

7. Dong S, Lau H, Chavarria C, Alexander M, Cimler A, Elliott JP, et al. (2019) Effects of Periodic Intensive Insulin Therapy: An Updated Review. Curr Ther Res Clin Exp. 90:61-67.

8. Pham H, Robinson-Cohen C, Biggs ML, Ix JH, Mukamal KJ, Fried LF, Kestenbaum B, Siscovick DS, de Boer IH. (2012) "Chronic Kidney Disease, Insulin Resistance, and Incident Diabetes in Older Adults." Clinical Journal of the American Society of Nephrology. 7;4: 588-594.

9. Spoto B, Pisano A, Zoccali C. (2016) "Insulin Resistance in Chronic Kidney Disease: A Systematic Review.” American Journal of Physiology-Renal Physiology. 311:6:F1087-1108.
This work is licensed under Creative Commons Attribution 4.0 License
Ready to submit your research? Choose Auctores and benefit from:

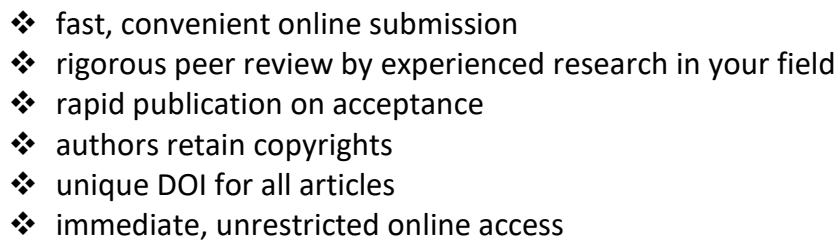

At Auctores, research is always in progress.

Learn more https://www.auctoresonline.org/journals/endocrinologyand-disorders 